\title{
Enfermedad de Gaucher: Un caso hondureño con confirmación genética
}

\author{
Gaucher Disease: A Honduran case with genetic confirmation \\ Flora Duarte, ${ }^{1}$ Rafael Mojica, ${ }^{2}$ Eda Sofía Cálix, ${ }^{3}$ \\ Carlos José Fajardo, ${ }^{4}$ Abigail Artica, ${ }^{5}$ Danilo Alvarado. ${ }^{6}$
}

\begin{abstract}
${ }^{1}$ Médica Especialista en Medicina Interna, sub-especialista en Hematología/Oncología; Centro de Cáncer Emma Romero de Callejas (CCERC), Tegucigalpa; ${ }^{2}$ Médico Especialista en Medicina Interna, sub-especialista en Hematología/Oncología; CCERC; Instituto Hondureño de Seguridad Social; Tegucigalpa; ${ }^{3}$ Médica Especialista en Salud Pública; CCERC; Tegucigalpa;

${ }^{4}$ Médico General; Candidato al grado de Maestría, Maestría en Epidemiología, Facultad de Ciencias Médicas, UNAH; CCERC; Tegucigalpa;

${ }^{5}$ Microbióloga con orientación en Análisis Clínico; CCERC; Tegucigalpa;

${ }^{6}$ Médico Especialista en Patología; Laboratorios Médicos; Tegucigalpa.
\end{abstract}

RESUMEN. Antecedentes: La enfermedad de Gaucher es causada por la acumulación del glicolípido glucosilceramidasa en el sistema macrofagocítico debido a la deficiencia de la enzima glucocerebrosidasa. Se han descrito tres formas clínicas de la enfermedad. La prevalencia mundial es de 1 caso en 40,000 100,000 nacimientos, aunque en poblaciones de ascendencia judío askenazi puede ser tan alta como 1 en 850 individuos. El diagnóstico definitivo se obtiene a través de observación directa de células Gaucher en médula ósea y la confirmación diagnóstica de las mutaciones en el cromosoma 1q21.31. Descripción del Caso: Femenina de 30 años con historia de 4 meses de hepatoesplenomegalia, leucopenia, neutropenia y trombocitopenia. Células de Gaucher se observaron en biopsia de médula ósea. Se confirmaron las variantes heterocigotas en los Exones 4 y 10 del Cromosoma 1q2. La paciente fue tratada con $5 \mathrm{mg}$ de ácido fólico y multivitaminas. Es candidata para Terapia de Reemplazo de la Enzima con imiglucerasa, sin embargo, éste no está disponible en Honduras por su alto costo. Paciente continúa en seguimiento 5 años después del diagnóstico. Conclusión: La incidencia en Honduras es desconocida. Otros casos podrían permanecer sin ser diagnosticados debido a la complejidad del diagnóstico en el primer nivel de atención. Las enfermedades metabólicas son un reto para el sistema de salud debido al acceso limitado a la atención médica de calidad y a la escasez de médicos especialistas capacitados. Una de las fortalezas de este caso es el estudio laboratorial completo que incluye confirmación genética de la enfermedad.

Palabras Clave: Enfermedad de Gaucher, Enfermedad por deficiencia de glucocerebrosidasa, Enfermedades Metabólicas, Glucosilceramidasa.
Recibido: 05-05-2020 Aceptado para publicación 28-06-2020

Dirección para correspondencia: Dr. Carlos José Fajardo,

Correo electrónico: cjfajardo92@gmail.com

Declaración de relaciones y actividades financieras y no financieras y conflictos de interés: ninguna.

DOI: https://doi.org/10.5377/rmh.v88i1.11592

\section{INTRODUCCIÓN}

La enfermedad de Gaucher (EG) es una enfermedad lisosomal por depósito del lípido glucosilceramidasa (glucocerebrosidase en inglés), el cual se acumula dentro de los macrófagos. Esto es causado por la deficiencia congénita de la $\beta$-glucocerebridasa, resultando en un daño multisistémico que compromete el sistema hematopoyético y óseo. ${ }^{1,2}$ Se han descrito clínicamente tres tipos de la enfermedad. El tipo más prevalente es el Tipo 10 también llamada forma no neuropática, la cual puede ser asintomática; pero que al manifestarse clínicamente puede presentar anemia, trombocitopenia, agrandamiento del bazo y alteraciones esqueléticas. La hepatoesplenomegalia puede causar síntomas mecánicos además de las manifestaciones pulmonares que también se han descrito en el Tipo 1. ${ }^{1}$ El tipo 2 de EG es excesivamente raro y es una forma neuropática aguda con un pronóstico grave que se caracteriza por un deterioro neurológico rápido y un mal pronóstico de vida a corto plazo. El tipo 3 de EG es caracterizado por un deterioro neurológico sub agudo en el cual los síntomas neurológicos tienen un comienzo más tardío y un mejor pronóstico en comparación al Tipo $2{ }^{1}$

Todos los tipos de EG son heredados de forma autosómica recesiva y tiene igual distribución entre sexos. La EG es una enfermedad que afecta a todas las etnias y cuya prevalencia mundial es de 1 caso en 40,000-100,000 nacimientos; sin embargo, en personas de ascendencia judía askenazi puede ser tan elevada como de 1 caso en 850 nacimientos., ${ }^{3,4}$ Las manifestaciones hematológicas de la EG incluyen anemia normocítica/normocrómica, leucopenia leve que en raras ocasiones se presenta con niveles menores a 1000 células/ $\mu \mathrm{L}$, una disminución en la quimiotaxis de neutrófilos y diversos grados de trombocitopenia. Las células de Gaucher son el sello patológico de la $E G$ y se encuentran principalmente en la médula ósea, bazo o en muestras de biopsia de hígado. Estas células tienen la apariencia típica de "papel arrugado". ${ }^{3}$ El gen de la glucosilceramidasa (GBA por sus siglas en inglés), localizado en el cromosoma 1q21, comprende 11 exones y 10 intrones. 
Un pseudogen altamente homólogo, GBAP por sus siglas en inglés, tiene la misma organización de exones e intrones como el gen funcional con una similitud de $96 \%$. Las mutaciones del GBA pueden aparecer en cualquiera de los exones (excepto en el 1) y puede causar EG. ${ }^{5}$

El tratamiento de EG históricamente ha consistido en el tratamiento sintomático de las citopenias, del hiperesplenismo y de las lesiones óseas. Desde épocas recientes, la Terapia de Reemplazo de la Enzima (ERT por sus siglas en inglés) que consiste en una suplementación intravenosa de la proteína deficiente Glucosilceramidasa, ${ }^{6}$ ha demostrado a través de ensayos clínicos aleatorizados y revisiones sistemáticas la mejora de las manifestaciones clínicas más evidentes de la enfermedad y es considerada ahora la primera línea de tratamiento para EG. ${ }^{6}$ La respuesta puede verse tan temprano como a los 6 meses para las manifestaciones hematológicas, en un año para los agrandamientos masivos del bazo y hasta 2 años para lesiones óseas. Como segunda línea de tratamiento, la Terapia de Reducción de Sustrato (SRT por sus siglas en inglés) es administrada de forma oral y su mecanismo de acción es inhibir la biosíntesis de la glucosilceramidasa, mitigando la acumulación patológica de glicolípidos y metabolitos en la EG. ${ }^{6}$

El curso y pronóstico de un paciente con EG es determinado por el tipo de enfermedad, el inicio temprano del tratamiento y el genotipo del paciente, siendo el primero el factor pronóstico más confiable. ${ }^{3}$ Luego de una revisión no sistemática de la literatura a través de PubMed, Google académico y en la Biblioteca Virtual en Salud se confirmó que este es el primer caso documentado de Enfermedad de Gaucher en Honduras y que además cuenta con confirmación genética del diagnóstico.

\section{DESCRIPCIÓN DEL CASO}

Se trata de paciente femenina de 30 años de edad, vista por primera vez en septiembre de 2014 en el Centro de Cáncer Emma Romero de Callejas (CCERC) en Honduras, con historia de 4 meses de evolución de esplenomegalia descrita como sensación de cuerpo extraño en el hipocondrio izquierdo. Las manifestaciones hematológicas comenzaron en el segundo semestre de 2013 durante su primer embarazo, en el cual se le practicó una cesárea debido a trombocitopenia. Durante los controles en el período post natal, nuevos hallazgos clínicos fueron encontrados incluyendo: hepatoesplenomegalia, leucopenia, neutropenia y confirmación de la trombocitopenia. Durante su primera atención en CCERC, en el examen físico se palpó esplenomegalia masiva de $24 \mathrm{~cm}$ que alcanza la región umbilical y hepatomegalia de $14 \mathrm{~cm}$, por debajo de reborde costal derecho.

El ultrasonido abdominal reportó hepatomegalia leve con predominio derecho, lóbulo derecho midió $17 \mathrm{~cm}$, con bordes regulares y definidos sin lesiones focales; además se observó marcada esplenomegalia difusa, con bordes regulares y definidos sin lesiones focales, midió aproximadamente $23 \times 15 \mathrm{~cm}$. En el hemograma completo, se encontró leucocitosis de 53.8 $10^{3} / \mathrm{mm}^{3}$, neutrofilia de $24.110^{3} / \mathrm{mm}^{3}(44.8 \%)$ y linfocitosis de $24.310^{3} / \mathrm{mm}^{3}(45.2 \%)$; hemoglobina de $12.9 \mathrm{~g} / \mathrm{dl}$ y plaquetas de 105 103/.mm . Pruebas especiales reportaron: $\beta 2$ microglo- bulina: $2,353.4 \mathrm{ng} / \mathrm{ml}$, eritrosedimentación: $45 \mathrm{~mm} / \mathrm{h}$, TSH 2.57 $\mathrm{UI} / \mathrm{ml}$. Dos meses después de primera evaluación, la paciente desarrolló leucopenia que persiste hasta la actualidad.

En el estudio de citometría de flujo de sangre periférica se evidenciaron dos grupos celulares de diferente tamaño y granularidad. CMF1790: se detectó una población celular que expresa los siguientes marcadores: CD20/CD45 positivo en 1.48\% (marcador linfoide B), HLA-DR positivo en 7.92\% (marcador de inmadurez), otros marcadores positivos: CD38 (9.83\%), IgG (4.43\%), CD15 (9.14\%), HVS (10.83\%). Otros marcadores linfoides $B(C D 10, C D 24, C D 43, C D 19)$, marcadores Linfoides $\mathrm{T}$ (CD2, CD8, CD3, CD56) marcadores de inmadurez, (CD34, TDT) y otros marcadores (CD42B, CD14, CD33, CD13, IgM, CD117) resultaron negativos.

Los exámenes de química sanguínea fueron: glucosa 91 $\mathrm{mg} / \mathrm{dl}$, urea: $49.4 \mathrm{mg} / \mathrm{dl}$, creatinina: $0.8 \mathrm{mg} / \mathrm{dL}$, calcio: $11.0 \mathrm{mg} /$ $\mathrm{dL}$, magnesio $2.2 \mathrm{mg} / \mathrm{dL}$, fósforo: $4.2 \mathrm{mg} / \mathrm{dL}$, ácido úrico: $2.8 \mathrm{mg} /$ $\mathrm{dL}$, proteínas totales: $9.3 \mathrm{~g} / \mathrm{dL}$, albúmina: $5.1 \mathrm{~g} / \mathrm{dL}, A S T(T G O)$ : 40.0 U/L, ALT (TGP): $16.0 \mathrm{U} / \mathrm{L}, \mathrm{LDH} 603.0 \mathrm{U} / \mathrm{L}$, fosfatasa alcalina: $118.0 \mathrm{U} / \mathrm{L}, \mathrm{GGT}: 10.0 \mathrm{U} / \mathrm{L}$, globulina: $4.2 \mathrm{~g} / \mathrm{dL}$.

Electroforesis de proteínas reportó: albúmina: $55.5 \%$ (valor de referencia: 59.8-72.4\%), alfa 1: 3.0\% (VR: 1.0-3.2), alfa 2: 9.1\% (VR: 7.4-12.6), beta: $8.1 \%$ (VR: 7.5-12.9), gamma: 24.3 (VR: 8.0-15.8). Se realizó una biopsia de médula ósea que reportó: Cilindro de médula ósea $70 \%$ células, $30 \%$ grasa, con un infiltrado de agregados histiocitarios que constituyen aproximadamente el $50 \%$ de la población celular. Histiocitos con núcleo excéntrico, citoplasma fibrilar (apariencia de papel arrugado) alternando con elementos residuales de serie eritroide, mieloide y megacariocítica (Figura 1 y Figura 2). Este tipo de histiocito es característico de la Enfermedad de Gaucher.

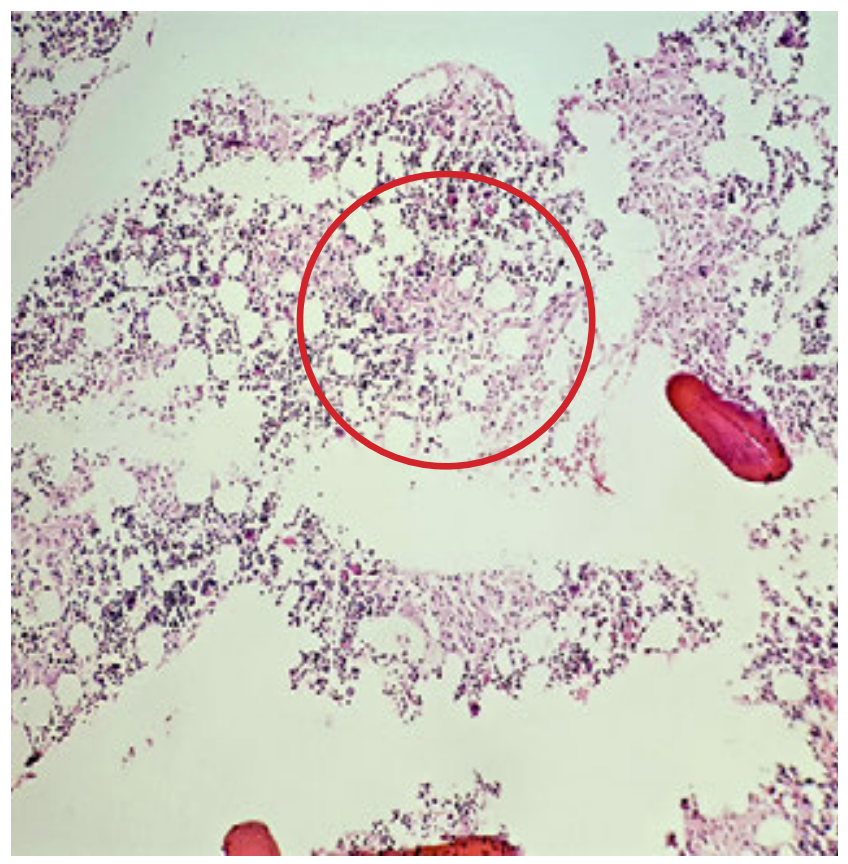

Figura 1. Biopsia de médula ósea, coloración Hematoxilina-Eosina, 40x. Se observa los agregados histiocitarios que reemplazan las células hematopoyéticas. Sección marcada se muestra con acercamiento en la Figura 2. 


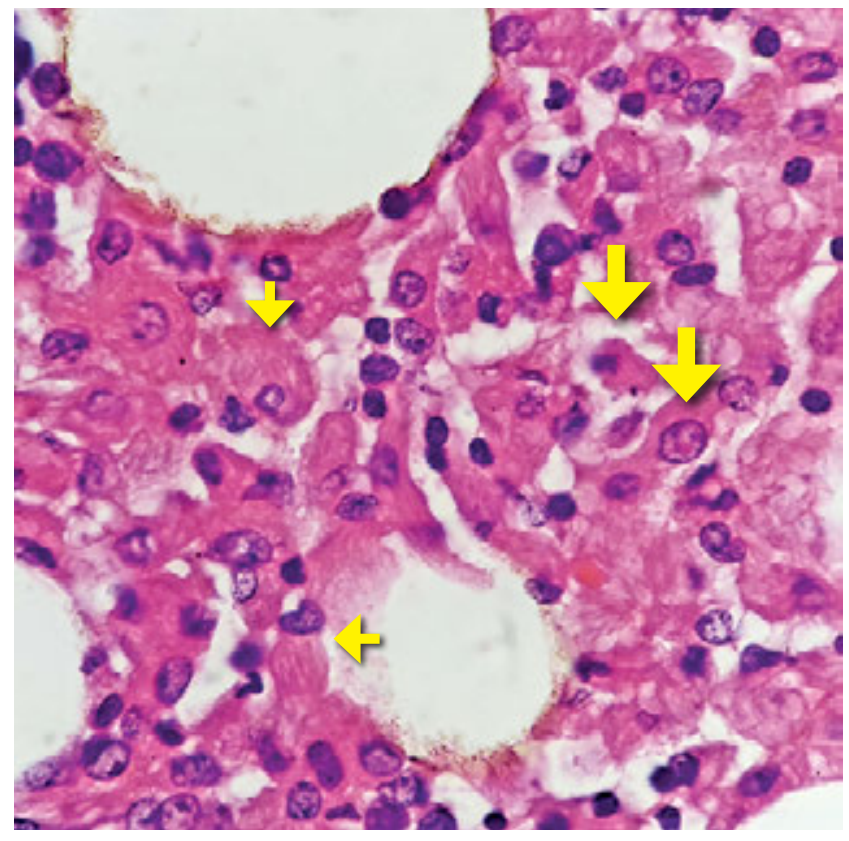

Figura 2. Biopsia de médula ósea, coloración Hematoxilina-Eosina, 100x. Se observan histiocitos con núcleos excéntricos (flechas) y citoplasma fibrilar con apariencia de papel arrugado (cabezas de flecha señalando zonas representativas) que es característico de la Enfermedad de Gaucher.

La paciente fue tratada con ácido fólico de $5 \mathrm{mg}$ y multivitaminas y continuó en seguimiento hasta enero 2016. En esa fecha, se enviaron muestras de sangre al Laboratorio de Errores Innatos del Metabolismo en Sao Pablo, Brasil para análisis genético. Se encontró una variante heterocigota en el Exon 4 ( $p$. V117A; HGMD acceso CM081644) y una variante heterocigota en Exon 10 (p. L483P: rs421016; HGMD acceso CM870010). Con estas mutaciones se confirmó el diagnóstico de Enfermedad de Gaucher y se clasificó como enfermedad tipo 1.

En septiembre 2017, después de 20 meses de seguimiento, la paciente regresó a CCERC luego de un segundo embarazo, el cual no tuvo ninguna complicación.

En junio 2019, radiografías de pelvis y de ambos brazos mostraron un leve descenso en la densidad ósea sin lesiones líticas ni fracturas. Actualmente, la paciente continúa en seguimiento con evaluaciones clínicas y laboratoriales. Últimos resultados de hemograma y química sanguínea: HGB: $11.9 \mathrm{~g} / \mathrm{dL}$, plaquetas 99 10/L, leucocitos $2.1610^{3}$, AST (TGO): $63.0 \mathrm{U} / \mathrm{L}$ y ALT (TGP): $35.6 \mathrm{U} / \mathrm{L}$. Paciente es candidata a recibir ERT o SRT pero debido a su alto costo este no está disponible en nuestro país.

\section{DISCUSIÓN}

La enfermedad de Gaucher es la esfingolipidosis más frecuente. ${ }^{7}$ Fue descrita por primera vez por Phillipe Gaucher en 1882. En su tesis, Gaucher describió la patología de la hasta entonces desconocida enfermedad, como una acumulación de células grandes con citoplasma estriado que afecta al bazo y al hígado y que impresionaba ser una proliferación no maligna. Fue hasta 1965, que la inhabilidad de degradar la glucosilceramidasa fue reconocida como el defecto primario. ${ }^{8}$
La incidencia mundial de EG oscila entre 1 caso por 40,000-100,000 nacimientos ${ }^{7}$; en Honduras la incidencia local es desconocida. En vista que la ascendencia de judíos Askenazi tiene una incidencia 1 caso por cada 850 nacimientos y que la evidencia histórica muestra que judíos askenazis se establecieron en Honduras, Centro América y otras regiones de América durante el periodo de colonización y durante la Segunda Guerra Mundial, ${ }^{9}$ se debe determinar la incidencia real de EG en Honduras. De existir esta carga genética en la población hondureña y por ser la EG una enfermedad de diagnóstico complejo, muchos casos en Honduras podrían permanecer sin diagnóstico. El caso presentado es una mujer mestiza la cual desconoce si tiene ascendencia judía.

Las enfermedades metabólicas representan un verdadero reto para nuestro sistema de salud debido al acceso limitado a los servicios médicos de calidad y a los pocos médicos especialistas con capacidad para diagnosticar este tipo de enfermedad. La confirmación de la mutación genética es todavía más complicada ya que tiene que hacerse en laboratorios en el extranjero. Una de las fortalezas de este reporte de caso es que, partiendo de conocimientos clínicos y anatomopatológicos, se logró obtener la confirmación del diagnóstico a través de la mutación específica de la enfermedad.

La EG tipo 1 tiene un espectro amplio de manifestaciones clínicas que incluyen varias combinaciones de esplenomegalia masiva, hepatomegalia, anemia, leucopenia, trombocitopenia y lesiones líticas en huesos. Estas manifestaciones clínicas pueden aparecer durante la infancia, la adultez temprana o incluso en edades avanzadas como la séptima u octava década de la vida. ${ }^{10,11}$ En el registro Francés de EG, Stirnemann et al encontraron que la mediana de edad de inicio de síntomas y del diagnóstico en una población de 562 pacientes fue de 15 y 22 años de edad, respectivamente para todos los tipos de enfermedad. ${ }^{12}$ En una revisión realizada por Charrow en 2000, reportó que la media de edad en pacientes en el Registro de Gaucher (del International Collaborative Gaucher Group, ICGG) es de 27.2 años de edad, siendo la mayoría (56\%) de los pacientes menores de 20 años. ${ }^{13}$ En un estudio de Cohorte realizado en Israel, el grupo de edad con la frecuencia más alta es de 35-44 años $(20 \%) .{ }^{14}$ En este caso, la edad de debut fue a los 30 años y las manifestaciones clínicas al diagnóstico consistieron en manifestaciones hematológicas y sin lesiones óseas. Se realizó el diagnóstico de EG cuando se observaron células de Gaucher en la médula ósea. Análisis de mutaciones genéticas confirmaron el diagnóstico a través de la identificación de las variantes patológicas en los Exones 4 y 10 del cromosoma 1q21, las cuales se han descrito como causantes de enfermedad.

Todos los pacientes requieren monitoreo, pero el manejo farmacológico no está indicado en todos los pacientes. ${ }^{7}$ En este caso, el manejo ha sido con ácido fólico y multivitaminas durante todo el seguimiento. Actualmente hay dos tipos específicos para EG: Terapia de reemplazo de la enzima (ERT por sus siglas en inglés) y terapia de reducción de sustrato (SRT por sus siglas en inglés). ${ }^{7}$ Estas opciones terapéuticas abordan directa 0 indirectamente la deficiencia de la enzima glucosilceramidasa y por ende la acumulación excesiva del lípido glucosilceramida. 
La complementación de la actividad deficiente de la enzima en el compartimento lisosomal de los macrófagos es la primera línea de tratamiento aceptada para la EG.7,15 Las enzimas recombinantes, tales como la imiglucerasa (Cerezyme $®$ ), taligucerasa alfa (Elelyso®) o la velaglucerasa alfa (VPRIV®) son administradas de forma intravenosa y han sido estudiadas a través de revisiones sistemáticas de Cochrane y se encontró que son bio-similares o no inferiores en cuanto a eficacia y seguridad durante el primer año de tratamiento. ${ }^{6}$ La respuesta a largo plazo de estos tratamientos aún no ha sido estudiada en ensayos prospectivos de larga duración. La SRT utiliza agentes reductores de sustrato que limitan la biosíntesis de glucosilceramida, por ende, aliviando la acumulación patológica de glicolípidos y metabolitos en la EG. ${ }^{15}$ La FDA (Food and Drug administration por sus siglas en inglés) ha aprobado dos SRTs orales para el tratamiento de EG: Miglustat (Zavesca®) en 2003 y Eliglustat tartrate (Cerdelga ${ }^{\circledR}$ ) en $2014 .{ }^{15}$ En caso de no recibir el tratamiento, se espera que la paciente presente una progresión de la enfermedad donde haya infiltración de la médula ósea y disfunción multiorgánica; además de mayor daño óseo por necrosis avascular, fracturas patológicas, aumento del dolor, progresión de la anemia, resultando en una reducción de la calidad de vida. ${ }^{16}$ En un estudio longitudinal realizado por Mistry $P$ et al, que analizó datos del ICGG, donde se evaluó la tasa de incidencia de osteonecrosis avascular en pacientes con EG, se encontró que entre los pacientes que iniciaron el tratamiento dentro de los dos primeros años posterior al diagnóstico, la tasa de incidencia de osteonecrosis avascular fue de 8.0 a 8.2 por cada 1,000 personas años. En cambio, la tasa de incidencia de osteonecrosis avascular entre los pacientes que iniciaron tratamiento con ERT más de 2 años después del diagnóstico se incrementó a 12.7 a 21.1 por cada 1000 persona-años. ${ }^{17}$ Además, éstos últimos presentaron mayor grado de anemia, menor conteo de plaquetas y mayor volumen esplénico. ${ }^{17}$ En el ICCG, la esperanza de vida de pacientes con ER se estima que es 7-9 años menor comparado con la población general, aunque con el tratamiento específico esta diferencia puede disminuirse en el futuro. ${ }^{18}$ En nuestro medio, el medicamento imiglucerasa (ERT) tiene un costo que se eleva hasta aproximadamente 36,000 dólares mensuales (según la casa farmacéutica Sanofi) cuando se administra en un horario con dosis altas y baja frecuencia (60 unidades/kg cada 2 semanas). El Centro de Cáncer Emma Romero de Callejas no cuenta con la capacidad económica para cubrir el costo de este tratamiento, sin embargo continuará la búsqueda del tratamiento en programas extranjeros compasivos.

\section{CONTRIBUCIONES}

FD y RM realizaron el diagnóstico clínico de la enfermedad, lideraron las decisiones terapéuticas y diagnósticas y concibieron la publicación del caso. EC y CF dieron seguimiento médico del caso y lideraron la redacción del manuscrito y la revisión de la literatura. DA realizó el diagnóstico anatomo-patológico y contribuyó sustancialmente con el análisis e interpretación de los hallazgos patológicos. AA participó en el análisis laboratorial y en la interpretación de los hallazgos genéticos. Todos los autores realizaron revisión crítica del contenido del manuscrito. Todos los autores aprobaron la versión a ser publicada y están de acuerdo a ser considerados responsables de todos los aspectos del trabajo.

\section{AGRADECIMIENTOS}

Se agradece a todo el personal técnico y de enfermería del CCERC por los servicios prestados durante el diagnóstico y seguimiento del caso. A la paciente que aceptó la publicación de su caso para dar a conocer las grandes necesidades que existen en nuestro país para tratar esta patología.

\section{REFERENCIAS}

1. Linari S, Castaman G. Clinical manifestations and management of Gaucher disease. Clin Cases Miner Bone Metab. 2015;12(2):157-64.

2. Bouayadi $O$, Lyagoubi $A$, Aarab A, Lamrabat $S$, Berhili A, Bensalah M, Seddik R. Gaucher Disease: an underdiagnosed pathology in the Eastern Moroccan Population. EJIFCC. 2019;1;30(1):82-87

3. Essabar L, Meskini T, Lamalmi N, Ettair S, Erreimi N, Mouane N. Gaucher's disease: report of 11 cases with review of literature. Pan Afr Med J. 2015;7;20:18.

4. Alaei MR, Tabrizi A, Jafari N, Mozafari H. Gaucher Disease: New Expanded Classification Emphasizing Neurological Features . Iran J Child Neurol. Winter. 2019;13(1):7-24.

5. Hruska KS, LaMarca ME, Scott CR, Sidransky E. Gaucher disease: mutation and polymorphism spectrum in the glucocerebrosidase gene (GBA). Hum Mutat. 2008;29(5):567-83.

6. Shemesh E, Deroma L, Bembi B, Deegan P, Hollak C, Weinreb NJ, Cox TM. Enzyme replacement and substrate reduction therapy for Gaucher disease. Cochrane Database Syst Rev. 2015;27;(3):CD010324.

7. Stirnemann J, Belmatoug N, Camou F, Serratrice C, Froissart R, Caillaud C, et al. A Review of Gaucher Disease Pathophysiology, Clinical Presentation and Treatments. Int J Mol Sci. 2017; 18(2):441.

8. European Working Group of Gaucher Disease. Gaucher disease [Internet] Germany: EWGGD; 2019. [consultado 23 septiembre 2019]. Disponible en: https://www.ewggd.com/gaucher-disease/
9. Villalobos Z, Villalobos J, Colina VL. Migraciones Judías en Centroamérica y el Caribe: Proyección Epidemiológica de la Enfermedad de Gaucher Jewish. VITAE Academia Biomédica Digital [Internet]. 2011[consultado 23 septiembre 2019];45. Disponible en: http://www.bioline.org.br/ request?va11004

10. Mistry PK, Belmatoug N, Vom Dahl S, Giugliani R. Understanding the natural history of Gaucher disease. Am J Hematol. 2015;90( Suppl 1):S6-11.

11. Baris HN, Cohen IJ, Mistry PK. Gaucher disease: the metabolic defect, pathophysiology, phenotypes and natural history. Pediatr Endocrinol Rev. 2014;12 Suppl 1(0 1):72-81.

12. Stirnemann J, Vigan M, Hamroun D, Heraoui D, Rossi-Semerano L, Berger $M G$, et al. The French Gaucher's disease registry: clinical characteristics, complications and treatment of 562 patients. Orphanet J Rare Dis. 2012;7:77.

13. Charrow J, Andersson HC, Kaplan P, Kolodny EH, Mistry P, Pastores G, et al. The Gaucher Registry: Demographics and Disease Characteristics of 1698 Patients With Gaucher Disease. Arch Intern Med [Internet]. 2000[consultado 12 octubre 2019];160(18):2835-43. Disponible en :https:// jamanetwork.com/journals/jamainternalmedicine/articlepdf/485470/ ioi90854.pdf.

14. Jaffe DH, Flaks-Manov N, Benis A, Gabay H, DiBonaventura M, Rosenbaum H, et al. Population-based cohort of 500 patients with Gaucher disease in Israel. BMJ Open. 2019;9(1):e024251. 
15. Barth BM, Shanmugavelandy SS, Tacelosky DM, Kester M, Morad SA, Cabot MC. Gaucher's disease and cancer: a sphingolipid perspective. Crit Rev Oncog. 2013;18(3):221-34.

16. Khan A, Hangartner T, Weinreb NJ, Taylor JS, Mistry PK. Risk factors for fractures and avascular osteonecrosis in type 1 Gaucher disease: a study from the International Collaborative Gaucher Group (ICGG) Gaucher Registry. J Bone Miner Res. 2012;27(8):1839-48.

17. Mistry PK, Deegan P, Vellodi A, Cole JA, Yeh M, Weinreb NJ. Timing of initiation of enzyme replacement therapy after diagnosis of type 1 Gaucher disease: effect on incidence of avascular necrosis. $\mathrm{Br} \mathrm{J}$ Haematol. 2009;147(4):561-70.

18. Weinreb NJ, Deegan P, Kacena KA, Mistry P, Pastores GM, Velentgas $\mathrm{P}$, et al. Life expectancy in Gaucher disease type 1. Am J Hematol. 2008;83(12):896-900.
ABSTRACT. Background: Gaucher Disease is a lipid storage disease caused by the accumulation of the glycolipid glucocerebroside in cells of the macrophage system due to a deficiency of glucocerebrosidase enzyme. Three major forms of the disease have been described. It has a worldwide prevalence of 1 case in 40,000-60,000 to 100,000 births, and in askenazi jewish heritage incidence is as high as 1 in 850 individuals. Definite diagnosis is obtained through direct observation of Gaucher Cells in bone marrow and genetic confirmation of the mutations in chromosome 1q21.31. Case Description: 30 years old female patient with a history of 4 months of hepato-splenomegaly, leukopenia, neutropenia and thrombocytopenia. Laboratory workup confirmed hematologic alterations. Gaucher Cells were observed in bone marrow biopsy. Exon 4 and Exon 10 heterozygous variants were confirmed in chromosome 1q2. Patient was treated with folic acid $5 \mathrm{mg}$ daily and multivitamins. She is candidate for Enzyme Replacement Therapy with imiglucerase, but this treatment is not available in Honduras due to its high price. Nowadays, follow up continues 5 years from clinical debut. Conclusion: Honduras' local incidence is still unknown and since GD is a complex diagnosis to be made in primary health care, most cases could remain undetected. Metabolic diseases are a real challenge in our health care system due to limited access to quality health care and few specialized physicians able to diagnose complex metabolic diseases. Genetic confirmation is not available. One of the strengths of this case report is a complete diagnostic work up including genetic confirmation.

Keywords: Gaucher Disease, Glucosylceramidase, Glucocerebrosidase deficiency disease, Metabolic disease. 